\title{
Development of Concurrent Object-oriented Logic Programming System to Intelligent Monitoring of Anomalous Human Activities
}

\author{
Alexei A. Morozov ${ }^{1,4}$, Abhishek Vaish ${ }^{2}$, Alexander F. Polupanov ${ }^{1,4}$, Vyacheslav E. Antciperov ${ }^{1}$, \\ Igor I. Lychkov ${ }^{3}$, Aleksandr N. Alfimtsev ${ }^{3}$ and Vladimir V. Deviatkov ${ }^{3}$ \\ ${ }^{1}$ Kotel'nikov Institute of Radio Engineering and Electronics of RAS, Mokhovaya 11, Moscow, Russia \\ ${ }^{2}$ Indian Institute of Information Technology, Deoghat, Jhalwa, Allahabad, UP, India \\ ${ }^{3}$ Bauman Moscow State Technical University, Vtoraya Baumanskaya 5, Moscow, Russia \\ ${ }^{4}$ Moscow State University of Psychology \& Education, Sretenka 29, Moscow, Russia
}

Keywords: Anomalous Human Activity, Intelligent Visual Surveillance, Logic Programming, Actor Prolog.

\begin{abstract}
A logic programming approach to the intelligent monitoring of anomalous human activity is considered. The main idea of this approach is in using of a first order logic for describing abstract concepts of anomalous human activity, i.e. brawls, sudden attack, armed attack, leaving object, loitering, pickpocketing, personal theft, immobile person, etc. We use the Actor Prolog concurrent object-oriented logic language and a state-of-the-art Prolog-to-Java translator for efficient implementation of logical inference on video scenes. A logical rules generation methodology is considered in relation to the analysis of anomalous human behaviour. The problem of creation of special built-in classes of Actor Prolog for the low-level video processing is discussed.
\end{abstract}

\section{INTRODUCTION}

Human activity recognition is a rapidly growing research area with important application domains including security and anti-terrorist issues (Aggarwal, 2011; Junior, 2010; Kim, 2010). Recently logic programming was recognised as a promising approach for dynamic visual scenes analysis (Filippou, 2012; Shet, 2011; O'Hara, 2008; Machot, 2011). The idea of a logic programming approach is in usage of logical rules for description and analysis of people activities. To approach the problem, knowledge about object co-ordinates and properties, scene geometry, and human body constraints is encoded in the form of certain rules in a logic programming language and is applied to the output of low-level object / feature detectors. There are several studies based on this idea. In (Filippou, 2012) a system was designed for recognition of socalled long-term activities (such as fighting and meeting) as temporal combinations of short-term activities (walking, running, inactive, etc.) using a logic programming implementation of the Event Calculus. The ProbLog state-of-the-art probabilistic logic programming language was used to handle the uncertainty that occurs in human activity recognition. An obvious merit of this approach is a high level of abstraction in describing human activity, but it may be too slow for the real time video processing. Mathematical semantics of probabilistic logical inference is also questionable. In (Shet, 2011) an extension of predicate logic with the bilattice formalism that permits processing of uncertainty in the reasoning was proposed. The VidMAP visual surveillance system that combines real time computer vision algorithms with the Prolog based logic programming had been announced by the same team. S. O'Hara (O'Hara, 2008) communicated the VERSA general-purpose framework for defining and recognising events in live or recorded surveillance video streams. According to (O'Hara, 2008), VERSA provides more advanced spatial and temporal reasoning than VidMAP and is based on SWI-Prolog. F.A. Machot et al. (Machot, 2011) have proposed real time complex audio-video event detection based on Answer Set Programming approach. The results indicate that this solution is robust and can easily be run on a chip.

Conventional approaches to human behaviour recognition include low-level and high-level ones. In this paper, we address the problem of the high-level 
semantic analysis of people activity. We use the Actor Prolog concurrent object-oriented logic programming language (Morozov, 1999, 2002, 2003, 2007, 2012) for implementation of the logical inference on video scenes. The Actor Prolog system includes a Prolog-to-Java translator that provides means for a high-level concurrent programming and a direct access to the low-level processing procedures written in Java.

In the case of simple human behaviour, a set of logic program rules can be created manually on the basis of a priori knowledge of the particular behaviour features, for example, speed of moving. In the case of complex spatio-temporal behaviour, a special methodology of generation of the logical rules is necessary.

We have described our first experiments in the area of human activity recognition in Section 2 . The problem of creation of special built-in classes of the Actor Prolog logic language for the low-level video processing is discussed in Section 3. A methodology of logical rules generation based on a hierarchy of fuzzy finite state automata is briefly considered in Section 4.

\section{LOGICAL ANALYSIS OF MANUALLY MARKED VIDEOS}

On the first stage of the research, we have performed several experiments on analysis of manually marked videos that is traditional approach in the area. The CAVIAR data sets (Fisher, 2007) were used. The CAVIAR data sets are annotated using the XMLbased Computer Vision Markup Language (CVML). The structure of CVML is simple enough, so we read it using the 'WebReceptor' built-in class of the Actor Prolog for XML / HTML parsing. The CVML annotations contain information about co-ordinates of separate persons and groups of persons in videos. So, our experiments have pursued the following goals:

1. To check whether the Actor Prolog system is fast enough to process videos in real time even without performing low-level analysis.

2. To check whether there is enough information about the positions of persons for accurate estimation of the velocity and the acceleration of separate personages in the video scene.

The latter issue is important because the accurate estimation of the velocity / acceleration opens a way for the recognition of so-called abrupt motions of objects (Filippou, 2012). This kind of motions is necessary for recognition of several longterm activities (such as fighting or sudden attack), though recognition of abrupt motions is not usually provided by standard low-level analysing procedures. The abrupt motions are not marked in the CAVIAR annotations as well.

An example of abrupt motion recognition is shown on the figure 1. A program written in Actor Prolog uses given co-ordinates of two persons to estimate the distance between them and the 2-nd derivative of the co-ordinates to detect abrupt motions of the persons.

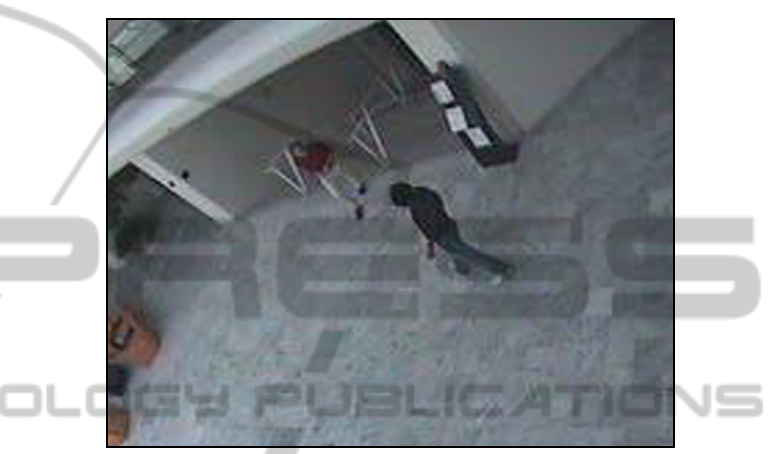

Figure 1: An example of CAVIAR video with a case of abrupt motions.

A logical rule describes an abnormal behaviour (fighting) as a conjunction of two conditions:

1. Several persons have met sometime and somewhere.

2. After that they implement abrupt motions.

The text of the logic program is not given here for brevity. After recognition of these two conditions, the logic programming system has decided that there was a case of scuffle and has indicated the fighting persons by a red rectangle (see figure 2).

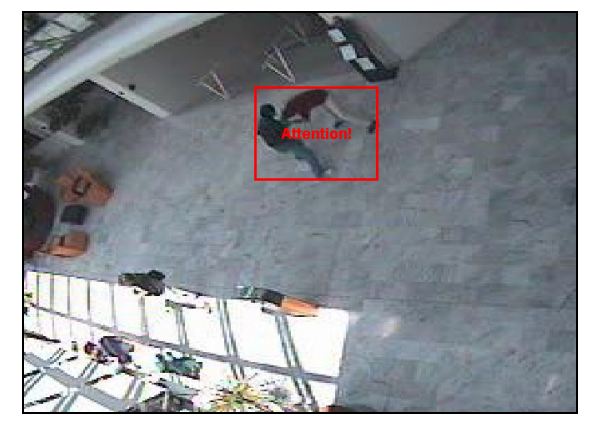

Figure 2: The logic programming system has recognised that two persons formed a group and were fighting.

This example demonstrates a possibility of recognition of video scenes semantics using the 
logical inference on results of the low-level recognition of separate objects; however one can see the following bottle-neck of the approach. Manually defined co-ordinates of the objects were used for estimation of their acceleration and nobody can guarantee that automatic low-level procedures will provide exact values of co-ordinates that are good enough for numerical differentiation. So, the discussion on the high-level recognition procedures is impossible without consideration of underlying low-level recognition methods.

The second issue of this example is whether it is useful to separate the recognition process into concurrent sub-processes implementing different stages of the high-level logical inference. Working intensity of different sub-processes is various. For example, the differentiation of co-ordinates requires more computational resources and another subprocess that implements recognition of people behaviour could wait for the results of differentiation.

\section{듵N드 AND TEC}

\section{ADVANCED LOGIC ANALYSIS OF VIDEO SCENES}

On the next stage of the research, we have implemented experiments on video analysis based on the automatically extracted information about coordinates and velocity of blobs in video scenes.

\subsection{Implementation of Base Low-level Video Processing Procedures}

A promising approach for implementation of the low-level recognition procedures in a logic language is usage of the OpenCV computer vision library and we are planning to link Actor Prolog with the JavaCV library that is a Java interface to OpenCV. Nevertheless, Java has enough standard tools to solve simple image processing / recognition problems and we have started our experiments with pure Java.

We have created low-level Java procedures that implement several basic recognition tasks:

1. Background subtraction;

2. Discrimination of foreground blobs;

3. Tracking of the foreground blobs over time;

4. Detection of interactions between the blobs.

The first experiments have demonstrated clearly that the exact estimation of an object velocity was impossible without taking into account the interactions of objects (see figure 3), because of edge effects of differentiation in the interaction points.

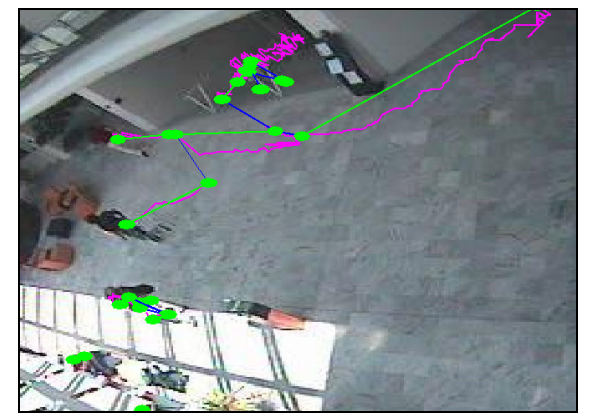

Figure 3: A low-level procedure discriminates trajectories (violet lines) of objects and moments of their interactions (green circle marks and blue links).

After implementation of the object interactions check, we have got tracks that were accurate enough to determine whether a person is walking or running. In the next section, we will describe an approach to lower boundary estimation of blob velocity and discuss its possible application to the detection of anomalous behaviour of people.

\subsection{A Fast Algorithm for Estimation of Object Velocity}

At this stage of research, we use standard method of recovering physical co-ordinates of objects in a scene, based on computing inverse matrix of projective transformation by co-ordinates of 4 defining points. A well-known disadvantage of this method is so-called ground plane assumption, that is, one cannot compute co-ordinates of body parts that are situated outside from a pre-defined plane. Usually, this pre-defined plane is a ground one and we can estimate properly the co-ordinates of person's shoes only. Generally speaking, this problem cannot be avoided in the framework of single camera approach, nevertheless, our idea is in usage of object velocity (but not co-ordinates) for the anomalous behaviour detection and this point is exploited in the following algorithm.

We consider simplified rectangle blobs describing moving objects in the scene (see example on figure 5). Co-ordinates of every corner of the blob are recovered using the inverse matrix of the projective transformation. Then, one compares the co-ordinates of corresponding corners of the blob in consecutive frames and calculates the first derivative of their co-ordinates. The idea is that only the corners situated in the ground plane give realistic estimations of velocity and other corners give 
greater values, because upper parts of body visually correspond to more distant points in the ground plane. So, we exploit this property of projective transformation and accept the lower boundary estimation of object velocity as a minimal value of velocities $\left(\mathrm{V}_{11}, \mathrm{~V}_{12}, \mathrm{~V}_{21}, \mathrm{~V}_{22}\right)$ of four blob corners:

$$
\mathrm{V} \approx \min \left(\operatorname{abs}\left(\mathrm{V}_{11}\right), \operatorname{abs}\left(\mathrm{V}_{12}\right), \operatorname{abs}\left(\mathrm{V}_{21}\right), \operatorname{abs}\left(\mathrm{V}_{22}\right)\right)
$$

Note, that the algorithm does not recover the direction of blob movement. The precision of the estimation of the blob velocity is not very high too, because of the approximate nature of the algorithm. Moreover, the automatic detection of blob shapes often produce illegal co-ordinates of blob corners because of common problems with shades, obstacles, digital noise etc, and this issue is an additional source of errors in the velocity estimation.

We have applied a median filtering to eliminate outliers in the velocity function. For instance, in the example on figure 4 , the 11 point median filter provides an estimation of blob velocity that is good enough for discrimination of running and walking persons in the scene.

We have implemented this algorithm of velocity estimation in the library of low-level methods of image analysis of the Actor Prolog system and use it in our experiments.

\subsection{Creation of a Built-in Class of Actor Prolog}

We have developed a special built-in class of the Actor Prolog language that uses formerly described low-level recognition procedures. The 'ImageSubtractor' class of Actor Prolog implements the following facilities:

1. Video frames pre-processing including 2Dgaussian filtering, 2D-median filtering, and background subtraction.

2. Recognition of moving blobs and creation of Prolog data structures describing the coordinates of the blobs in every moment.

3. Recognition of tracks of blob motions and creation of Prolog data structures describing the co-ordinates and the velocity of the blobs. The tracks are divided into separate segments; where segment ends there are points of interaction between the blobs.

4. Recognition and ejection of immovable and slowly moving objects. This feature is based on simple fuzzy inference on the attributes of the tracks (the co-ordinates of the tracks and the average velocities of the blobs are considered).

5. Recognition of connected graphs of linked tracks of blob motions and creation of Prolog data structures describing the co-ordinates and the velocity of the blobs.

We consider two tracks as linked ones if there are interactions between the blobs of these tracks. In some applications, it is useful to eject tracks of immovable and slowly moving objects from the graphs before further processing of the video scenes.

\subsection{An Example of Anomalous Behaviour Detection}

Let us consider an example of logical inference on video. The input of the logic program written in Actor Prolog is the Fight_RunAwayl sample provided by the CAVIAR team (the sequence of JPEG files is used). The program will use no additional information about the content of the video scene, but only co-ordinates of 4 defining points in the ground plane (the points are provided by CAVIAR). The total text of the logic program is not given here for brevity; we will discuss only the program structure and main stages of data analysis.

The logic program creates two concurrent processes with different priorities (see Morozov, 2003 for details about Actor Prolog model of asynchronous concurrent computations). The first process has higher priority and implements video data gathering. This process reads JPEG files and sends them to the instance of the 'ImageSubtractor' predefined class that implements all low-level processing of video frames. The sampling rate of the video is 25 frames per second, so the process loads a new JPEG file every 40 milliseconds.

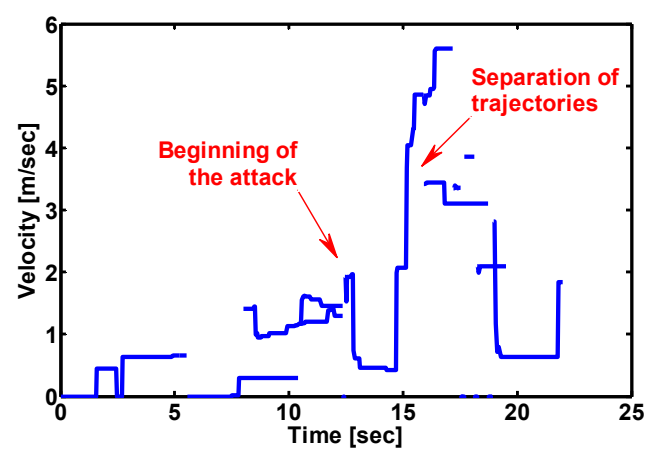

Figure 4: An example of estimation of velocities of blobs in a visual scene (see figure 3 ). The $\mathrm{X}$-axis denotes time in seconds and the Y-axis denotes lower boundary estimation of blob velocities $(\mathrm{m} / \mathrm{sec})$. One can recognise walking persons (before the beginning of the attack) and running persons (after the separation of the trajectories of persons) on the diagram. 
The second concurrent process implements logical analysis of collected information and outputs results of the analysis. The analysis of video frames requires more computational resources, but it does not suspend the low-level analysis, because the second process has less priority. The analysis includes extraction of blobs, tracking of the blobs over time, detection of interactions between the blobs, creation of connected graphs of linked tracks of blobs, and estimation of average velocity of blobs in separate segments of tracks (see figure 4). This information is received by the logic program in a form of Prolog terms describing the list of connected graphs.

The 'ImageSubtractor' class uses the following data structures for describing connected graphs of tracks (note, that the DOMAINS, the PREDICATES, and the CLAUSES program sections in Actor Prolog have traditional meaning developed in the Turbo / PDC Prolog systems):

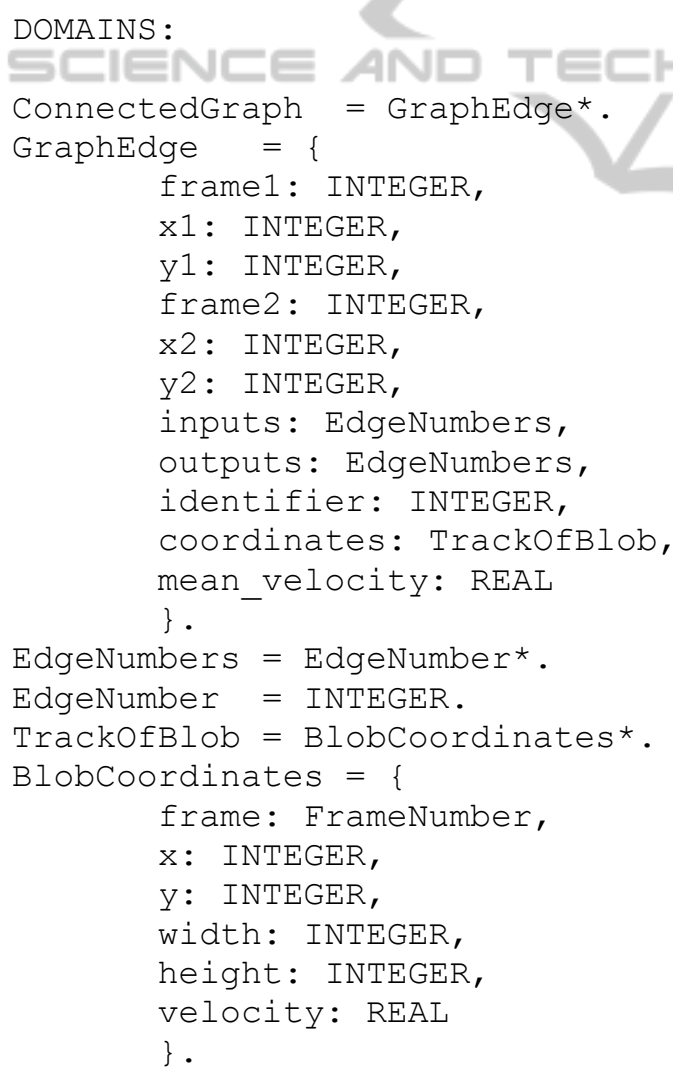

That is, connected graph is a list of underdetermined sets (Morozov, 1999) denoting separate edges of the graph. Every edge is directed and has the following attributes: numbers of front and last frames (frame1, frame2), co-ordinates of front and last points $(\mathrm{x} 1, \mathrm{y} 1, \mathrm{x} 2, \mathrm{y} 2)$, a list of edge numbers that are predecessors of the edge (inputs), a list of edge numbers that are followers of the edge (outputs), the identifier of corresponding blob (an integer identifier), a list of sets describing the co-ordinates and the velocity of the blob in different moments of time (coordinates), and an average velocity of the blob in this edge of the graph (mean_velocity).

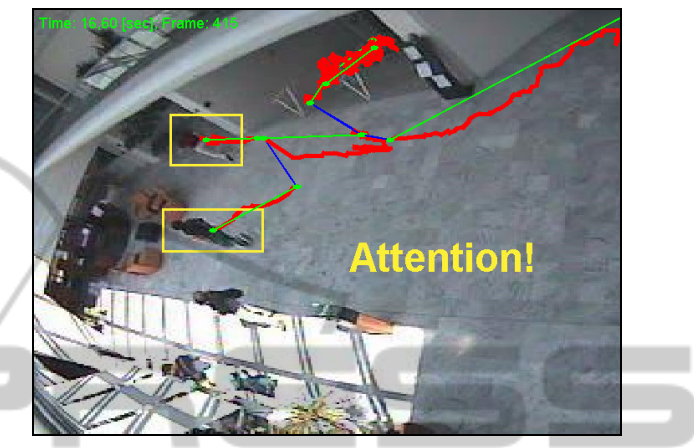

Figure 5: A logical inference has found a possible case of sudden attack in the graph of blob trajectories. Rectangle blobs are depicted by yellow lines, blob trajectories are depicted by red lined, moments of interactions between blobs are depicted by green circles and blue links.

The logic program checks the graph of tracks and looks for the following pattern of interaction among several persons: if two or more persons met somewhere in the scene, and one of them has walked (not run) before this meeting, and one of them has run (not walked) after this meeting, the program considers this scenario as a kind of a lam and a probably case of a sudden attack or a theft. So, the program alarms if this kind of sub-graph is detected in the total graph of tracks. In this case, the program draws all tracks of the graph under consideration in red and outputs the "Attention!" warning in the middle of the screen (see figure 5).

One can describe formally the concept of a lam using defined connected graph data type.

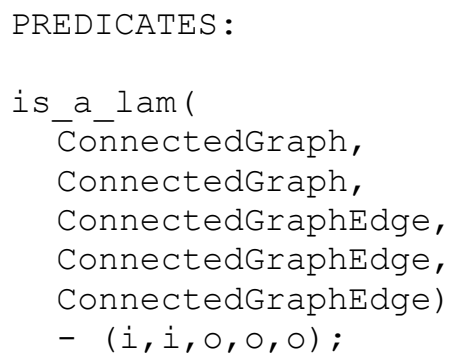

We will define the is_a_lam $(G, G, P 1, E, P 2)$ predicate with the following arguments: $G$ - a graph to be analysed (the same data structure is used in the 
first and the second arguments), $E$ - an edge of the graph corresponding to probable incident, $P 1-$ an edge of the graph that is a predecessor of $E, P 2$ - an edge that is a follower of $E$. Note that $G$ is an input argument of the predicate and $P 1, E$, and $P 2$ are output ones. Here is Actor Prolog program code with brief explanations:

\section{CLAUSES :}

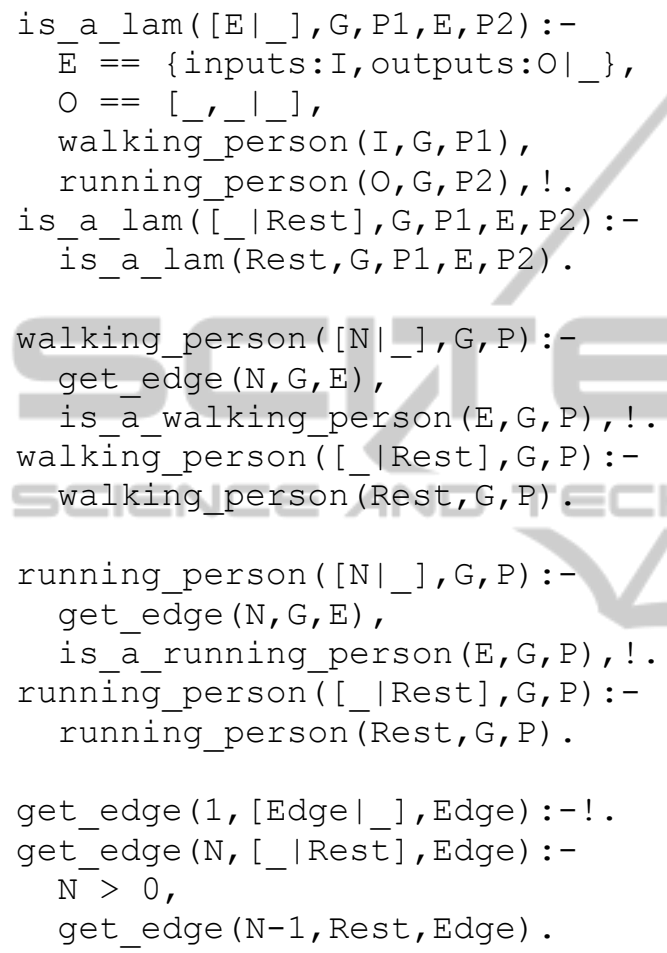

In other words, the graph contains a case of a lam if there is an edge $E$ in the graph that has a predecessor $P 1$ corresponding to a walking person and a follower $P 2$ that corresponds to a running person. It is requested also that $E$ has more than one follower (it is a case of branching in the graph).

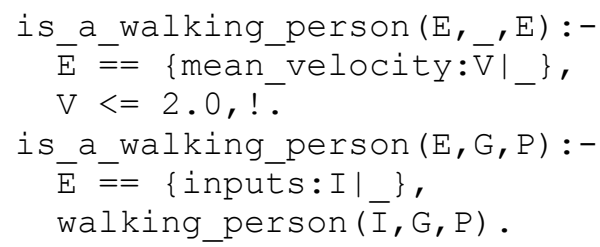

That is, the graph edge corresponds to a walking person if the average blob velocity in this edge is less or equal to $2 \mathrm{~m} / \mathrm{sec}$, or the edge has a predecessor that corresponds to a walking person.

The graph edge corresponds to a running person if the average velocity in this edge is more or equal to $3 \mathrm{~m} / \mathrm{sec}$, or the edge has a follower corresponding

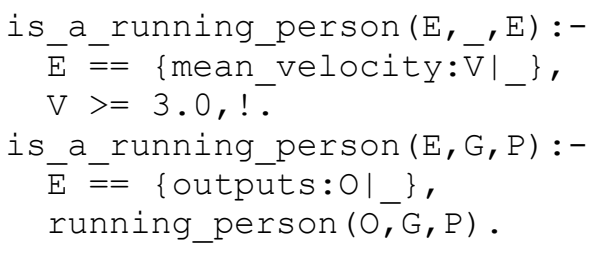

to a running person.

Note that aforementioned rules use plain numerical thresholds to discriminate walking and running persons for brevity. Better discrimination could be provided by a kind of a fuzzy check, which can be easily implemented using arithmetical means of standard Prolog.

The example illustrates the possible scheme of a logic program implementing all necessary stages of video processing including video information gathering, low-level image analysis, high-level logical inference on the video scene, and reporting the results of intelligent visual surveillance.

\section{LOGICAL RULES GENERATION METHODOLOGY}

A logical rules generation methodology based on a hierarchy of fuzzy finite state automata was introduced in (Devyatkov, 2005). Concepts and notations described in (Devyatkov, 2005) should be modified according to the specified problem domain. Let $T=\left\{t_{i} \mid t_{i} \in N\right\}$ be a discrete set of time instances with constant intervals $\Delta t=t_{i+1}-t_{i}$ between consecutive time instances. Let $\left[t_{s}, t_{e}\right]=\left\{t \mid t_{s} \leq t \leq t_{e}\right\}$ be a time interval $T$. Assume that each $0^{\text {th }}$ level feature (human speed and position for our example) of each moving object $\theta$ from set $\left\{\theta^{1}, \theta^{2}, \ldots, \theta^{l}\right\}$ at time instance $t$ can take on a value $y_{i_{0}}\left(\theta_{t}\right), i_{0} \in\left\{1, \ldots, m_{0}\right\}$, called feature sample. Samples tuple $Y_{i_{0}}\left[\theta_{t_{s}}, \theta_{t_{e}}\right]=\left\langle y_{i_{0}}\left(\theta_{t_{s}}\right), \ldots, y_{i_{0}}\left(\theta_{t_{e}}\right)\right\rangle$, $i_{0} \in\left\{1, \ldots, m_{0}\right\}$ of a single $0^{\text {th }}$ level feature taken at several consecutive time instances $t_{s}, \ldots, t_{e}$ during the $\left[t_{s}, t_{e}\right]$ time interval is called a trend.

Let us consider the following situation. Two persons walk along perpendicular lines towards their intersection. While person $A$ is far from intersection, person $B$ slows down waiting for person $A$. When person $A$ enters intersection, person $B$ accelerates and runs into person $A$.

In order to formalise the persons' behaviour, 
appropriate features should be specified. Let persons $A$ and $B$ walk along perpendicular lines with intersection point $O$. Let $x O y$ be a rectangular coordinate system such that $O x$ axis corresponds to the line person $A$ walks along, $O y$ axis corresponds to the line person $B$ walks along (figure 6). For the purpose of simplicity, each person is considered as a rectangle, thus the person co-ordinates are rectangle centroid co-ordinates. Since persons move strictly along the co-ordinate axes, the current position of persons $A$ and $B$ can be determined by single coordinates $y_{s}\left(\theta_{t}^{A}\right)$ and $y_{s}\left(\theta_{t}^{B}\right)$ measured along corresponding co-ordinate axes. The persons' position co-ordinates $y_{s}\left(\theta_{t}^{A}\right), y_{s}\left(\theta_{t}^{B}\right)$ are treated as first features. The persons' speed values $y_{v}\left(\theta_{t}^{A}\right)$, $y_{v}\left(\theta_{t}^{B}\right)$ are treated as second features.

One should specify observation time interval $\left[t_{s}, t_{e}\right]=\left\{t \mid t_{s} \leq t \leq t_{e}\right\}$ along with time instances $t_{i} \in\left[t_{s}, t_{e}\right]$ the feature samples to be taken at. In practice, observation time interval is determined with some physical considerations in mind, e.g. maximum time required for passing the observed path segment; time instances are determined by the surveillance equipment. Particular parameter values assignment is outside of the scope of this paper.

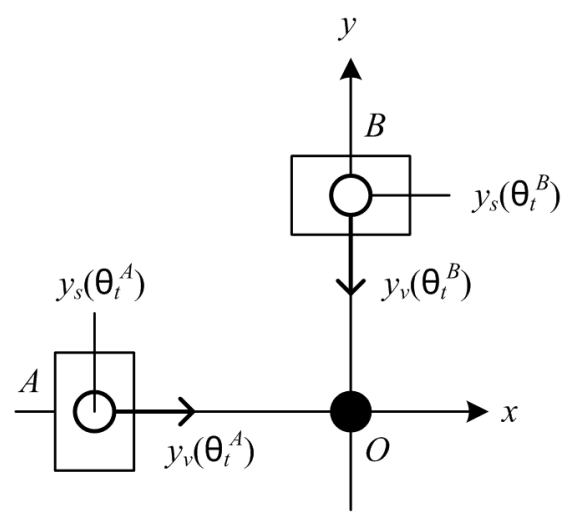

Figure 6: Two persons in rectangular co-ordinate system.

Linguistic variables are specified and behaviour template models are composed according to (Devyatkov, 2005). Let position $\left(\theta^{A}\right), \operatorname{speed}\left(\theta^{A}\right)$ and position $\left(\theta^{B}\right), \quad \operatorname{speed}\left(\theta^{B}\right)$ be linguistic variables that describe position and speed of persons $A$ and $B$. Linguistic variables position $\left(\theta^{A}\right)$ and position $\left(\theta^{B}\right)$ take on linguistic values $\operatorname{far}\left(\theta^{X}\right)$, near $\left(\theta^{X}\right)$, and inside $\left(\theta^{X}\right)$. Linguistic variables
$\operatorname{speed}\left(\theta^{A}\right)$ and $\operatorname{speed}\left(\theta^{B}\right)$ take on linguistic values $\operatorname{high}\left(\theta^{X}\right)$ and $\operatorname{low}\left(\theta^{X}\right)$.

Figures 7 and 8 present fuzzy sets corresponding to linguistic values $\operatorname{far}\left(\theta^{X}\right), \operatorname{near}\left(\theta^{X}\right), \operatorname{inside}\left(\theta^{X}\right)$ and $\operatorname{high}\left(\theta^{X}\right), \operatorname{low}\left(\theta^{X}\right)$.

Fuzzy sets shown on figures 7 and 8 are used to compose first level template automata $M_{p o s\left(\theta^{X}\right)}$, $M_{\text {speed }\left(\theta^{A}\right)}$ and $M_{\text {speed }\left(\theta^{B}\right)}$ that describe position and speed of persons $A$ and $B$.

Automaton $M_{p o s\left(\theta^{X}\right)}$, shown as graph on figure 4 , determines sequence of linguistic values $\left[\operatorname{far}\left(\theta^{X}\right), \quad \operatorname{near}\left(\theta^{X}\right), \quad\right.$ inside $\left.\left(\theta^{X}\right)\right]$ of linguistic variable position $\left(\theta^{X}\right)$. The automaton graph is based on a chain of allowed states $b_{11}-b_{12}-b_{13}$ corresponding to linguistic values of the determined sequence. $b_{11}$ is the initial state (marked with input arrow on figure 9) and $b_{13}$ is the final state (marked with output arrow on figure 9) of the automaton. State transitions are specified as follows. Provided with input linguistic value corresponding to the current state, automaton retains its current state. Provided with input linguistic value corresponding to the next allowed state, the automaton moves to that state. Automaton moves to denied state $b_{14}$ in case of input linguistic value confronting to allowed sequence of the automaton. Having entered the denied state once, the automaton cannot leave this state. Automata $M_{\text {speed }\left(\theta^{A}\right)}$ and $M_{\text {speed }\left(\theta^{B}\right)}$ are presented as graphs on figures 10 and 11 . Automaton graphs are different since behaviour of person $A$ differs from behaviour of person $B$.

The second level template automaton should be composed in order to describe joint persons' behaviour. Let condition $\left(\theta^{A}, \theta^{B}\right)$ be a linguistic variable that takes on linguistic values $\operatorname{safe}\left(\theta^{A}, \theta^{B}\right)$, warning $\left(\theta^{A}, \theta^{B}\right)$, and unsafe $\left(\theta^{A}, \theta^{B}\right)$ specified with the following relations:

$$
\begin{gathered}
\text { safe }\left(\theta^{A}, \theta^{B}\right)=\left[\operatorname{pos}\left(\theta_{t}^{A}\right)=\operatorname{far}\left(\theta^{A}\right)\right] \wedge \\
{\left[\operatorname{pos}\left(\theta_{t}^{A}\right)=\operatorname{far}\left(\theta^{A}\right)\right]} \\
\operatorname{warning}\left(\theta^{A}, \theta^{B}\right)=\left(\left[\operatorname{pos}\left(\theta_{t}^{A}\right)=\operatorname{near}\left(\theta^{A}\right)\right] \wedge\right. \\
\left.\left[\operatorname{speed}\left(\theta_{t}^{B}\right)=\operatorname{high}\left(\theta^{B}\right)\right]\right) \vee \\
\left(\left[\operatorname{speed}\left(\theta_{t}^{A}\right)=\operatorname{high}\left(\theta^{A}\right)\right] \wedge\left[\operatorname{pos}\left(\theta_{t}^{B}\right)=\operatorname{near}\left(\theta^{B}\right)\right]\right) \\
\text { unsafe }\left(\theta^{A}, \theta^{B}\right)=\left[\operatorname{pos}\left(\theta_{t}^{A}\right)=\operatorname{inside}\left(\theta^{A}\right)\right] \wedge \\
{\left[\operatorname{pos}\left(\theta_{t}^{B}\right)=\operatorname{inside}\left(\theta^{B}\right)\right]}
\end{gathered}
$$




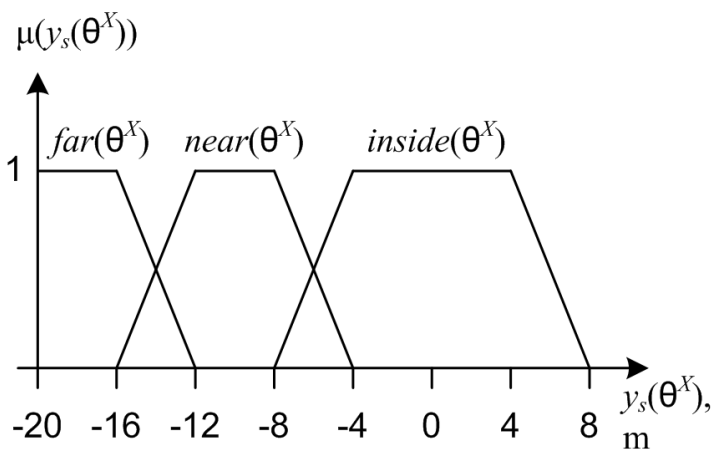

Figure 7: Fuzzy sets, corresponding to linguistic values $\operatorname{far}\left(\theta^{X}\right), \operatorname{near}\left(\theta^{X}\right)$, and inside $\left(\theta^{X}\right)$.

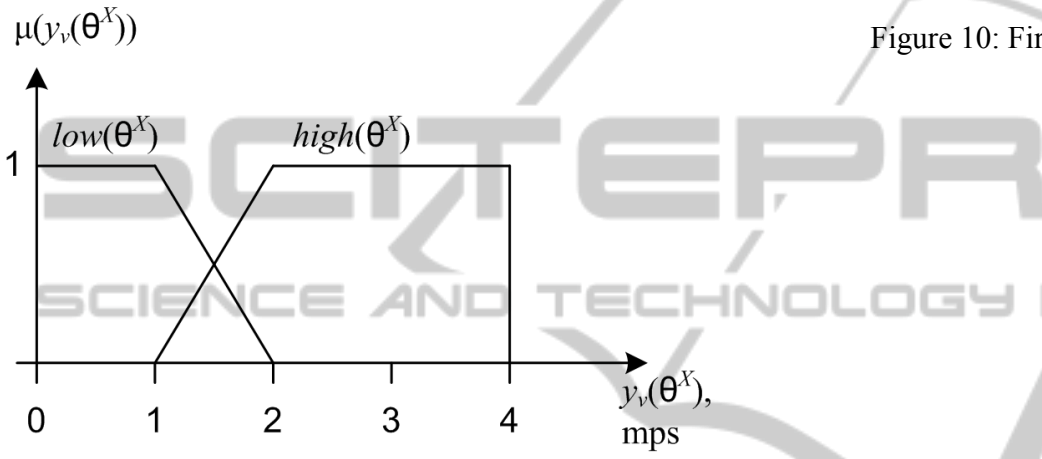

Figure 8: Fuzzy sets, corresponding to linguistic values $\operatorname{low}\left(\theta^{X}\right)$ and $\operatorname{high}\left(\theta^{X}\right)$.

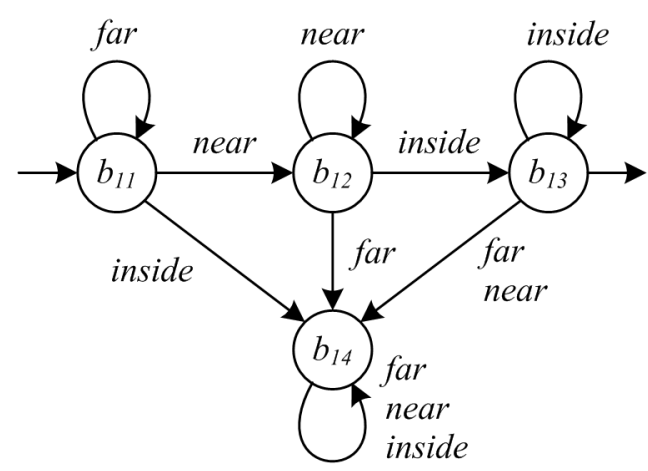

Figure 9: First level automaton $M_{p o s}\left(\theta^{X}\right)$ graph.

Each linguistic value of a linguistic variable condition $\left(\theta^{A}, \theta^{B}\right)$ corresponds to a composite fuzzy set, determined on a multidimensional domain. Domain of the composite fuzzy set is a Cartesian product of domains of fuzzy sets involved into the corresponding relation (Devyatkov, 2005). According to equation (1) domain of linguistic value $\operatorname{safe}\left(\theta^{A}, \theta^{B}\right)$ is equal to

$\operatorname{dom}\left[\operatorname{safe}\left(\theta^{A}, \theta^{B}\right)\right]=\operatorname{dom}\left[\operatorname{near}\left(\theta^{A}\right)\right] \times \operatorname{dom}\left[\operatorname{far}\left(\theta^{B}\right)\right]$, where $\operatorname{dom}[E]$ is domain of fuzzy set $E ; \times$ is Cartesian product.

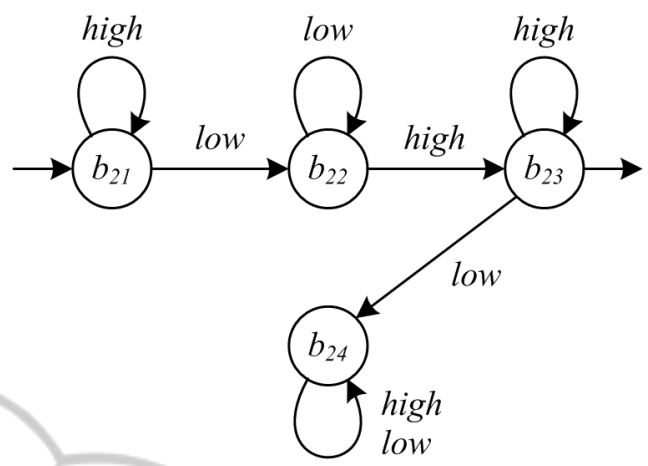

Figure 10: First level automaton $M_{\text {speed }}\left(\theta^{A}\right)$ graph.

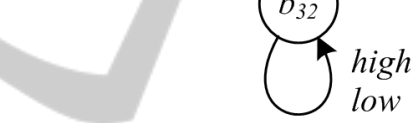

Figure 11: First level automaton $M_{\text {speed }}\left(^{B}\right)$ graph.

Membership function of composite fuzzy set can be expressed through membership functions of fuzzy sets involved into the corresponding relation according to the following rules:

$$
\begin{gathered}
a=b \wedge c \rightarrow R_{a}\left(y_{b}, y_{c}\right)=\min \left\{R_{b}\left(y_{b}\right), R_{c}\left(y_{c}\right)\right\} \\
a=b \vee c \rightarrow R_{a}\left(y_{b}, y_{c}\right)=\max \left\{R_{b}\left(y_{b}\right), R_{c}\left(y_{c}\right)\right\} \\
a=\neg b \rightarrow R_{a}\left(y_{b}\right)=1-R_{b}\left(y_{b}\right),
\end{gathered}
$$

where $a, b, c$ are fuzzy sets; $R_{E}$ is membership function of fuzzy set $E$, determined on domain $\operatorname{dom}[E] ; \quad y_{b} \in \operatorname{dom}[b], \quad y_{c} \in \operatorname{dom}[c]$ are feature values from corresponding domains.

According to equation (4), membership function of composite fuzzy set, specified on a conjunction of two fuzzy sets, is equal to minimum value among membership functions of its composing fuzzy sets.

Value of membership function numerically expresses the goodness of current feature values for a distinct linguistic value. Linguistic variable $\operatorname{condition}\left(\theta^{A}, \theta^{B}\right)$ is set to linguistic value with the most membership function for current feature 
values.

Second level template automaton $M_{\text {condition }\left(\theta^{A}, \theta^{B}\right)}$ that describes the joint persons' behaviour is shown on figure 12 .

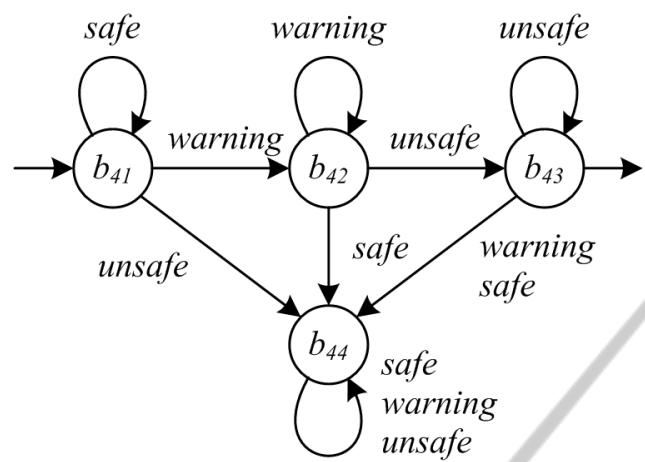

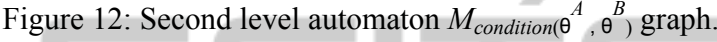

The computational methodology scheme is presented on figure 13. It includes five units for linguistic variables evaluation and processing, arranged in two levels. Each unit compute value of corresponding linguistic variable and inputs it to a corresponding automaton.

Situation recognition is implemented as follows. Initially, all first and second level automata are reset to their initial states. Then feature samples for consecutive time instances $t_{i} \in\left[t_{s}, t_{e}\right]$ are passed by turn into the first level units for evaluation and processing of the first level linguistic variables. The first level units compute values of linguistic variables and pass them into the second level unit for evaluation and processing of the second level linguistic variable condition $\left(\theta^{A}, \theta^{B}\right)$. During operation, the first and the second level automata may change their states. Situation is recognised if all first and second level automata have moved to their final states after all feature samples have been processed. Situation is not recognised if one automaton at least has not moved to its final state.

The computational methodology scheme based on fuzzy finite automata can easily be converted to a logic program, using standard techniques of transforming finite state machines into the logic programs (Bratko, 1986).

\section{CONCLUSIONS}

A prospective approach for implementing the logic programming to the problem of intelligent monitoring of people activity is a translation from a concurrent object-oriented logic programming language to Java. Our study has demonstrated that the Actor Prolog logic programming system is suitable for this purpose and provides essential separation of the recognition process into concurrent sub-processes implementing different stages of highlevel analysis.

A specialised built-in class of the Actor Prolog language implementing simple pre-processing of

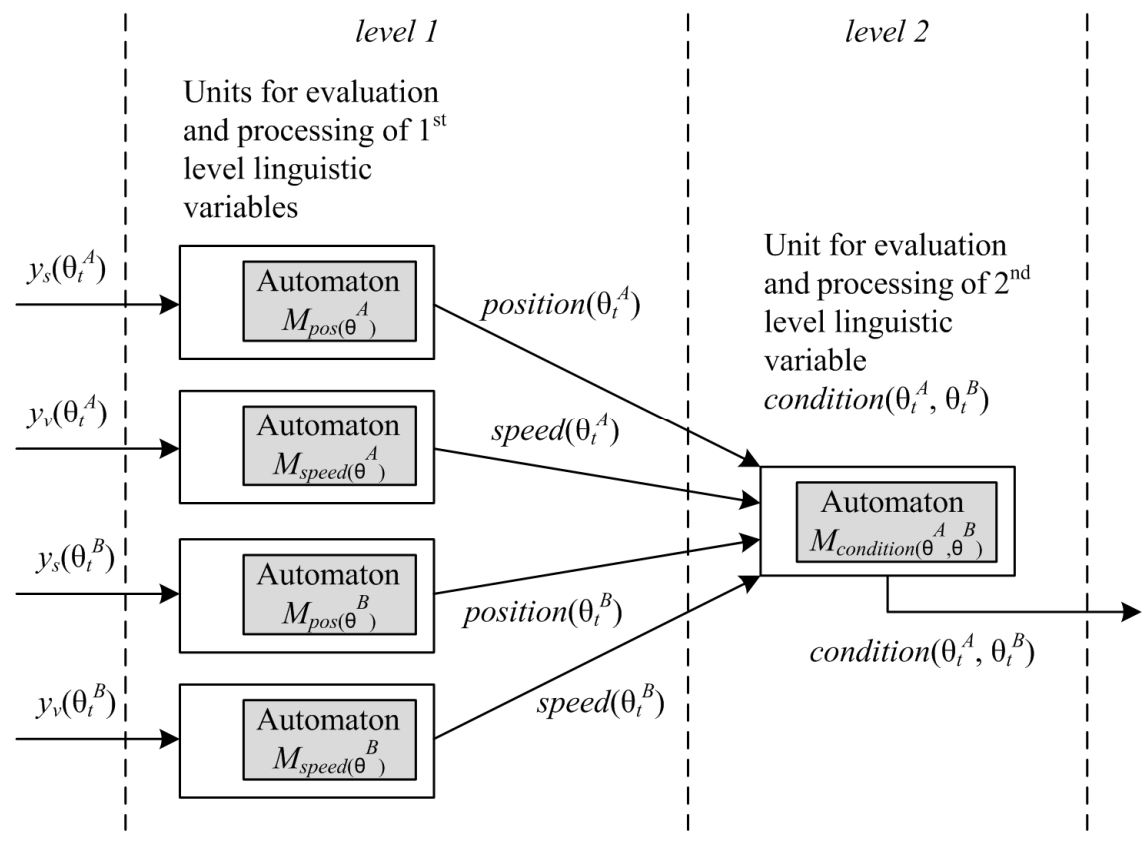

Figure 13: The computational methodology scheme. 
video data and low-level analysis of video scenes concerning the problem of intelligent monitoring of people activity was demonstrated. We have implemented a simple analysis of videos based on automatically extracted information on the coordinates and velocities of blobs in the video scene. It was shown that robust recognition of abrupt motions is impossible without accurate low-level recognition of body parts (face, hands). This is a subject of further studies.

An extension of the Actor Prolog logic programming system to advanced algorithms of lowlevel video processing and to investigations of new possibilities at the level of logical analysis is discussed. It is supposed to complete a prototype of an open source Java library for studying logical description and analysis of people behaviour in order to facilitate researches in the field of intelligent monitoring of anomalous people activity.

A logical rules generation methodology is proposed for situation analysis in the environment of moving objects. A formal method for representing situations using hierarchy of fuzzy finite state automata was considered. Future work will include comprehensive testing of the proposed methods on massive datasets and development of fully automatic method for situation representation using real feature trends.

\section{ACKNOWLEDGEMENTS}

We acknowledge a partial financial support from the Russian Foundation for Basic Research, No 13-07-92694, and Department of Science and Technology, Govt. of India, No DST-RFBR P-159.

\section{REFERENCES}

Aggarwal, J. K., Ryoo, M. S. 2011. Human Activity Analysis: A Review. ACM Computing Surveys (CSUR), 43 (3), April.

Bratko, I. 1986. Prolog Programming for Artificial Intelligence. Addison-Wesley Publishing Company.

Devyatkov, V. V. 2005. Multiagent hierarchical recognition on the basis of fuzzy situation calculus. Vestnik, Journal of the Bauman Moscow State Technical University, Natural Science \& Engineering, 2005, pp. 129-152.

Filippou, J., Artikis, A., Skarlatidis, A., Paliouras, G. 2012. A Probabilistic Logic Programming Event Calculus. Computing Research Repository, abs/1204.1851. [Online] Available from: http://arxiv.org/abs/1204.1851.
Fisher, R. 2007. CAVIAR Test Case Scenarios. The EC funded project IST 2001 37540. [Online] Available from: http://homepages.inf.ed.ac.uk/rbf/CAVIAR/.

Junior, J., Musse, S., Jung, C. 2010. Crowd analysis using computer vision techniques. A survey. IEEE Signal Processing Magazine, September, pp. 66-77.

Kim, I. S., Choi, H. S., Yi, K. M., Choi, J. Y., Kong, S. G. 2010. Intelligent Visual Surveillance - A Survey. International Journal of Control, Automation, and Systems, 8 (5), pp. 926-939.

Machot, F. A., Kyamakya, K., Dieber, B., Rinner, B. 2011. Real Time Complex Event Detection for Resource-Limited Multimedia Sensor Networks. In: Workshop on Activity monitoring by multi-camera surveillance systems (AMMCSS), pp. 468-473.

Morozov, A. A. 1999. Actor Prolog: an Object-Oriented Language with the Classical Declarative Semantics. In: IDL'99, Paris.

Morozov, A. A. 2002. On Semantic Link between Logic, Object-Oriented, Functional, and Constraint Programming. In: MultiCPL'02, Ithaca, pp. 43-57.

Morozov, A. A. 2003. Logic Object-Oriented Model of Asynchronous Concurrent Computations. Pattern Recognition and Image Analysis, 13 (4), pp. 640-649.

Morozov, A. A. 2003. Development and Application of Logical Actors Mathematical Apparatus for Logic Programming of Web Agents. In: ICLP 2003 Proceedings. Springer-Verlag, LNCS 2916, pp. 494-495.

Morozov, A. A. 2007. Operational Approach to the Modified Reasoning, Based on the Concept of Repeated Proving and Logical Actors. In: CICLOPS, Porto, pp. 1-15.

Morozov, A. A. 2007. Visual Logic Programming Method Based on Structural Analysis and Design Technique. In: ICLP 2007 Proceedings. Springer-Verlag, LNCS 4670, pp. 436-437.

Morozov, A. A. 2012. Actor Prolog to Java translation. In: IIP-9, Montenegro, Budva. Moscow: Torus Press, pp. 696-698. In Russian.

O'Hara, S. 2008. VERSA - Video event recognition for surveillance applications. M.S. thesis, University of Nebraska at Omaha.

Shet, V., Singh, M., Bahlmann, C., Ramesh, V., Neumann, J., Davis, L. 2011. Predicate Logic Based Image Grammars for Complex Pattern Recognition. International Journal of Computer Vision, 93 (2), June, pp. 141-161. 